\title{
Structural synthesis of serial platform manipulators
}

\author{
Rasim Alizade *, Cagdas Bayram, Erkin Gezgin \\ Mechanical Engineering Department, Izmir Institute of Technology, Urla 35437, Izmir, Turkey \\ Received 4 July 2005; received in revised form 5 May 2006; accepted 16 May 2006 \\ Available online 18 July 2006
}

\begin{abstract}
In this paper, structural synthesis of serial platform manipulators is considered. Serial platform manipulators are created according to the development of the platforms and closed loops. Also a new structural formula of mobility of parallel Cartesian platform robot manipulators is presented. Structural synthesis of serial platform manipulators with lower and higher kinematic pairs with respect to their structures are also examined. Structural synthesis of parallel Cartesian platform robot manipulators is also introduced. History of structural formulas DOF are presented as a table with equations, authors, years and some commentaries. New and revised methods for structural synthesis of serial platform manipulators and parallel Cartesian platform robot manipulators are illustrated along with examples.
\end{abstract}

(c) 2006 Elsevier Ltd. All rights reserved.

Резюме

в предстабленной работе рассматриьаются вопросы структурного синтеза платформенных манипулятороb последобателыной структуры. Создание платформенных манипудятороb посдедоватедыной структуры осноbана на разьитии платформ и замкнутых контуроb. в работе предстабпена ноbая структурная формула, а также рассматрибаются bопросы структурного синтеза платформенных манипулятороb последобателыной структуры с низщими и бысшими парами с точки зрения их стркуктуры. История структурны формул степени подьижности предстарлена $\mathrm{b}$ bиде таблицы с урабнениями, авторами, годами и некоторыми коментариями. Нобые и пересмотренные методы структурного синтеза платформенных манипулятороb последобателыной структуры иллюстрируются различыми примерами.

(C) 2006 Elsevier Ltd. All rights reserved.

Keywords: Structural groups; Mobility loop-legs equation; Serial platform manipulators; Parallel Cartesian manipulators

\footnotetext{
* Corresponding author. Tel.: +90 232750 6544; fax: +90 2327507890.

E-mail addresses: rasimalizade@iyte.edu.tr, alizada_rasim@hotmail.com (R. Alizade).
} 


\section{Introduction}

Structural synthesis problem is the first step in the design of new robot manipulators and the fundamental concept in robot design. The mobility of robotic mechanical system describes the number of actuators needed to define the location of end-effectors. It is important that the mobility or the degrees of freedom of robot manipulators $(M>1)$ indicates the number of independent input parameters to solve the problem of all the configuration of robots or a kinematic chain with several actuators. If mobility of the kinematic chain is equal to zero $(M=0)$ and cannot be split into several structural groups, we will get a simple structural group. Combining the simple structural groups with actuators, we can get serial or parallel robot manipulators. IFToMM terminology defines "manipulator that controls the motion of its end-effector by means of at least two kinematic chains going from the end-effector towards the frame" as parallel manipulator. In parallel manipulators, two platforms cannot be connected by kinematic pairs to each others.

Serial platform manipulators control the motion of the platforms by means of at least two platforms, which are connected by kinematic pairs, and other kinematic chains going from the platforms towards the frame. Several connections of the links in series for gripping and the controlled movement of objects are called serial manipulators. Combination of serial and parallel manipulators gives hybrid robot manipulators. Complex robot manipulators consist of independent loops with variable general constraint $(\lambda=2,3,4,5,6)$.

The history of works about the number of independent loops was done by Euler [1]. Then in the second half of the 19th century, the first structural formulas of mechanisms by Chebyshev [2], Sylvester [3], Grübler [4], Somov [5], Gokhman [6] were created. As shown in Table 1, in the mobility equations we can find concepts of the number of independent loops $(L)$, degrees of freedom or mobility of mechanisms $(M)$, the loop motion parameters $(\lambda)$, the number of joints $(j)$, number of moving links $(n)$, number of mobility of kinematic pairs $(f)$ and independent joint constraints $(s)$, number of passive mobilities $\left(j_{\mathrm{p}}\right)$, and the number of overclosing constraints $(q)$. To describe and compare the structural formulas and the parameters in structural analysis and synthesis of robotic mechanical system, the unique key controlling parameters are used as shown in Table 1.

Furthermore, the concepts of the structural formulas and simple structural groups were developed in the first half of the 20th century by Koeings [7], Assur [8], Muller [9], Malushev [10], Kutzbach [11], Kolchin [12], Artobolevskii [13], Dobrovolskii [14]. As shown in Table 1, some new concepts in the problem of structural analysis and synthesis of mechanisms had been reached as number of screw pairs $\left(S_{c}\right)$, simple structural groups with zero mobility $(M=0)$, number of kinematic pairs with $i$ class ( $p_{i}$, where $i$ is the number of joint constraint), number of links with variable length $\left(n_{v}\right)$, variable general constraint $\left(\lambda_{K}\right)$, and the family of the elementary closed loop $\left(d_{K}=6-\lambda_{K}\right)$.

During the second half of the 20th century, the productive results to find general methods for determination of the mobility of any mechanisms had been obtained by Moroshkin [15], Voinea and Atanasiu [16], Paul [17], Rössner [18], Boden [19], Ozol [20], Waldron [21], Manolescu [22], Bagci [23], Antonescu [24], Freudenstein and Alizade [25], Hunt [26], Herve [27], Gronowicz [28], Davies [29], Agrawal and Rao [30], Dudita and Diaconescu [31], Angeles and Gosselin [32], Alizade [33], McCarthy [34]. In the calculation of mechanism mobility, the following new parameters were used (Table 1), as the rank of linear independent loop equations or the order of the equivalent screw system of the closed loop $(r)$, relative displacements of the joint $(m)$, number of independent, scalar, differential loop-closure equations $\left(\lambda_{K}\right)$, the rank of the coefficient matrix $(r(j))$, finite dimensional vector space $(d(v))$, new formula of the number of independent loops $\left(L=j_{B}-B-c_{b}\right.$, where $j_{B}$ is the total number of joints on the platforms, and $c_{B}$ is the total number of branches between moving platforms and $B$ is the number of moving platforms), serial open chains connecting to ground or total number of robot legs $\left(c_{l}\right)$, and the degree of constraint of the platform $(U)$. It should be noted that, branches are the kinematic chains that connects mobile platforms to each others, and legs are the kinematic chains that connects mobile platforms to the fixed frame.

In the beginning of 21st century, further developments of robotic science has arisen the interest in scientific investigations. New parameters in the structural formulas describing the real physical essences should be created in the new investigations and be more suitable for the use in practice in new subjects. In this direction, last investigations can be introduced as Huang [35], Alizade and Bayram [36], Gogu [37], Alizade, Bayram and Gezgin (formulas \#38 in Table 1). In the calculation of degrees of freedom of mechanisms, the following new parameters are used in the structural formulas, as a new formulation of the number of independent loops 
Table 1

Formulas for structural analysis and synthesis

\begin{tabular}{|c|c|c|c|}
\hline & Equations & Authors & Commentary \\
\hline 1 & $\begin{array}{l}L=j-l+1 \\
l \text { is the number of links; } j \text { is } \\
\text { the number of joints }\end{array}$ & Euler [1] & $L$ is the number of independent loops \\
\hline 2 & $\begin{array}{l}3 l_{m}-2 j-1=0 \\
0<j-j_{m}<1+\frac{1}{2} l \\
j_{m}>l-3 \quad l_{m}=n=l-1\end{array}$ & Chebyshev [2] & $\begin{array}{l}\text { Equation for planar mechanisms } \\
\text { with } 1 \text { dof } \\
j_{m} \text { is the number of moving joints } \\
l_{m}=n \text { is the number of moving links }\end{array}$ \\
\hline 3 & $\begin{array}{l}3 l-2 j-4=0 \\
j=n-1\end{array}$ & Sylvester [3] & $\begin{array}{l}\text { Equation for planar mechanisms } \\
\text { with } 1 \text { dof }\end{array}$ \\
\hline 4 & $\begin{array}{l}\text { (a) } M=3 l-2 j-3 \\
\text { (b) } 3 l-2 j-4+q=0 \\
\text { (c) } 2 l-j-3=0 \\
\text { (d) } 3 l-2 j-4+q-C=0 \\
\text { (e) } 5 H-6 l+7=0 \\
\text { or } M=6(l-1)-5 p_{1}\end{array}$ & Grübler [4] & $\begin{array}{l}M \text { is mobility of mechanisms. dof } \\
\text { depends from the rank of functional } \\
\text { determinant }(r=3,2) \\
\text { (a) dof for planar mechanisms } \\
\text { (b) Equation for kinematic chains } \\
\text { with revolute } R \text { and prismatic } P \text { pairs } \\
\text { (c) Equation for plane mechanisms } \\
\text { just with prismatic } P \text { pairs } \\
\text { (d) Equation for kinematic chains } \\
\text { with revolute, prismatic and cam pairs } \\
\text { (e) dof of spatial mechanisms with } \\
\text { helical joints }\end{array}$ \\
\hline
\end{tabular}

$q$ is the number of overclosing constraints

$p_{1}$ is the one mobility joints

$C$ is the number of cam pairs

$H$ is the number of helical joints

$\begin{array}{ll}\text { (a) } l-(\lambda-1)(v+1)=2 & \text { Somov [5] }\end{array}$

(b) $l+q+\sum K_{u}-(\lambda-1)(v+1)=2$

(c) $M=(l-1)+\sum f_{i}-j-5 L+q$

$l=5 v+7, \lambda=6, v=L-1$,

$\sum K_{u}=j_{\mathrm{p}}-1$

$j_{\mathrm{p}}$ is the passive mobilities in the joints

$f_{i}$ is the mobility of kinematic pairs

(a) $\lambda(l-1)-S=1$

Gokhman [6]

$S=\sum(\lambda-i) f_{i}$ is the total number

of independent joint constraints
(b) $\sum f_{i}-\lambda L=1$
(c) $\lambda(j-L)-S=1$

$(\lambda-1) S_{\mathrm{s}}-\lambda l+(\lambda+1)=0$

$M=\lambda n-(\lambda-1) S_{\mathrm{s}}$

$S_{\mathrm{s}}$ is the number of screw pairs

(a) Equation for plane $(\lambda=3)$ and spatial $(\lambda=6)$ mechanisms $(M=1)$

(b) Equation for plane and spatial mechanisms $(M=1)$

(c) Somov's universal structural formula $\lambda$ is the number of independent parameters describing the position of rigid body (general constraint parameter)

(a) Equation for plane and spatial mechanisms $(M=1)$

(b) Loop mobility criterion $(M=1)$

(c) Equation for mechanisms $(M=1)$ Eqs.

(a) and (c) gives Euler's equation

Mobility equations for spatial mechanisms (similar to Gokhman equation)

Equation for simple structural groups

Equation for kinematic chains with screw pairs (similar to M. Grubler equation)

Universal Somov-Malushev's mobility equation $n_{v}$ is the number of links with variable length 
Table 1 (continued)

\begin{tabular}{|c|c|c|c|}
\hline & Equations & Authors & Commentary \\
\hline 11 & $\begin{array}{l}M=\lambda(l-j-1)+\sum_{i=1}^{j} f_{i} \\
M=\lambda(l-1)+\sum_{i=1}^{j}(\lambda-i) f_{i}\end{array}$ & Kutzbach [11] & Other form of universal mobility equation \\
\hline \multirow[t]{2}{*}{12} & $\begin{array}{l}M=3(l-1)-2(P+R+K)-p_{2} \\
P \text { is the number of prismatic pairs }\end{array}$ & Kolchin [12] & $\begin{array}{l}\text { Structural formula for planar mechanisms. } \\
K \text { is the number of higher pairs with pure } \\
\text { roll or pure slippage }\end{array}$ \\
\hline & $R$ is the number of revolute pairs & & $\begin{array}{l}p_{2} \text { is the number of higher pair with rolling } \\
\text { and slipping }\end{array}$ \\
\hline 13 & $M=6 n-\sum_{i=1}^{j} S_{j}+\sum_{K=1}^{L} d_{K}+q$ & Artobolevskii [13] & $\begin{array}{l}\text { Other form of universal mobility equation first } \\
\text { time in mobility equation, it is used variable } \\
\text { general constraint as variable number of } \\
\text { independent close loops family }\end{array}$ \\
\hline & $\begin{array}{l}d_{K}=6-\lambda_{K} \text { is the family of the } \\
\text { elementary closed loop or the number }\end{array}$ & & $\lambda_{K}$ is the variable general constraint \\
\hline
\end{tabular}

Dobrovolskii [14]

Moroshkin [15]

(b) $M=\sum_{i} i p_{i}-\sum_{\lambda} \lambda L_{\lambda}$

$i=1, \ldots, 5 \quad \lambda=2, \ldots, 6$

(c) $L=j-n$

16

17

18

$21 \quad M=F-r$
$M=\sum_{i=1}^{j} f_{i}-\sum_{K=1}^{L} r_{K}-j_{\mathrm{p}}$

$\sum_{i=1}^{j} f_{i}$ is the total number

dof of joints with revolute, prismatic and helical joints

$L=j-l+1$

$M=\sum_{i=1}^{j} f_{i}-6(j-l+1)$

$M=\sum_{i=1}^{j} f_{i}-6(j-l+1)-3(j-l+1)$

(a) $M=\sum_{i=1}^{j} f_{i}-6 L+q$

(b) $M=\sum_{i=1}^{j} f_{i}-3 L+q$

(c) $M=2(l-1)-j+q$

(d) $M=j-2 L+q$

$F$ is the relative freedom between links

Voinea and

Atanasiu [16]

Paul [17]

Rössner [18]

Boden [19]

Ozol [20]

Waldron [21]

Manolescu [22]

Bagci [23]

$M=6(l-1)-\sum_{i=1}^{5}(6-i) f_{i}$

$+\sum_{K=1}^{L} d_{K}+\sum q-\sum j_{\mathrm{p}}$

$M=\left(6-d_{a}\right)(l-1)-\sum_{i=1}^{5}\left(i-d_{a}\right) p_{i}$
Other form of universal structural formula

(a) Structural equation of system with the integrable joining

(b) Equation of the dof with variable general constraint

(c) Number of independent close loops $r=\lambda$ is the rank of linear independent loop

Mobility equation of a complex mechanisms $1 \leqslant r_{K} \leqslant 6$ is the rank of screw system

Using formula \#1, it was created topological condition of criterion for the degree of constraint of plane kinematic chains

The mobility equation taking into consideration Euler's formula \#1

Mobility equation, consisting from the planar and the spatial loops

(a), (b), and (c) mobility equations for variable general constraint, as $\lambda=6,3,2$ with excessive constraints

(d) mobility equation for cylindrical mechanisms $(\lambda=2)$

Mobility equation of closed loop $r$ is the order of the equivalent screw system of the closed loop

Mobility equation with the parameter of the family of the elementary closed loop

Mobility equation to calculate dof of motion in a mechanism similar to equation \#13 by adding parameter $j_{\mathrm{p}}$

Mobility formulas with different values for the motion coefficient $\lambda$ (formula \#14)

(continued on next page) 
Table 1 (continued)

\begin{tabular}{lll}
\hline Equations & Authors & Commentary \\
\hline 25 & Freudenstein and & Mobility equations without exception \\
(a) $M=\sum_{i=1}^{E} m_{i}-\sum_{K=1}^{L} \lambda_{K}$ & Alizade [25] & (a) and (b) mobility equations are used for \\
(b) $M=\sum_{i=1}^{j} f_{i}-\sum_{K=1}^{L} \lambda_{K}$ & & $\begin{array}{l}\text { mechanisms which contain mixed independent } \\
\text { loops with variable general constraint. } \\
\text { (c) and (d) Mobility equations of mechanisms with }\end{array}$ \\
(c) $M=\sum_{i=1}^{E} m_{i}-\lambda L$ & & the same number of independent, scalar loop closure \\
& & equations in each independent loop. \\
(d) $M=\sum_{i=1}^{j} f_{i}-\lambda L$ & & $\lambda_{K}$ is the number of independent, scalar, differential \\
loop closure equations & $\lambda$ is the dof of space where the mechanism operates
\end{tabular}

displacement variable

$m_{i}$ is the relative displacements of the joints

$f_{i}$ is the relative joint motion

when $m_{i}$ correspond in 1:1 with dof in joints

$M=\lambda(l-j-1)+\sum_{i=1}^{j} f_{i}$

$M=\lambda(l-1)-\sum_{i=1}^{j}\left(\lambda-f_{i}\right)$

$M=\sum \lambda_{K}-\sum_{K=1}^{L-1} \sum_{j=K+1}^{L} F_{K j}$

$F_{K j}$ is the mobility of the joints that

is common between any two loops $K$

and $j$, and the mobility of the joints in the

$L$ loops can be counted once or twice

$$
\begin{aligned}
M= & \sum_{K \overline{\bar{N}}_{1}}^{L} \lambda_{K}-\sum_{K=1}^{L-1} \sum_{j=K+1}^{L} F_{K j} \\
& +\sum_{i=1}^{1} \frac{1}{2}\left(\tilde{n}_{i}^{2}+\tilde{n}_{i}-2\right) F_{\tilde{n} i} \\
& +\sum_{i=1}^{N_{2}} \frac{1}{2}\left(n^{2}-3 n_{i}+2\right) F_{n i}
\end{aligned}
$$

(a) $M=\sum_{i=1}^{j} f_{i}^{e}-\sum_{K=1}^{L} \lambda_{K}^{e}$

(b) $M=\sum_{K=1}^{L} \lambda_{K}-\sum_{j}\left(L_{\mathrm{comj}}-1\right) f_{\mathrm{comj}}^{e}$

$L_{\text {comj }}$ is the number of loops

with common joint $j$

$f_{\text {comj }}^{e}$ is the active degree of mobility

of the $j$ th common joint

$$
M=\operatorname{nullity}(J)
$$

$\operatorname{nullity}(J)=\mathrm{d}(v)-r(J)$

$\mathrm{J}$ is the Jacobian matrix;

$r(J)$ is the rank of

the Jacobin matrix; $d(v)$ is the

finite dimensional vector space $v$
(a) $L=j_{B}-B-c_{b}$
(b) $M=\sum_{i=1}^{E} m_{i}-\lambda\left(j_{B}-B-c_{b}\right)+q-j_{\mathrm{p}}$
(c) $M=\sum_{i=1}^{j} f_{i}-\lambda\left(j_{B}-B-c_{b}\right)+q-j_{\mathrm{p}}$
(d) $\sum_{i=1}^{j} f_{i}=\lambda\left(j_{B}-B-c_{b}\right)$

\section{Hunt [26]}

Herve [27]

Gronowicz [28]

Davies [29]

Agrawal and Rao [30]

Dudita and

Diaconescu [31]

Angeles and Gosselin [32]

Alizade [33]
Mobility equation coming from Eq. (25d) using Eq. (1)

Mobility formula based on the algebraic group structure of the displacement set

Mobility equation for multi loop kinematic chains
Mobility equations similar to Eq. (\#15a) $r$ is the rank of the coefficient matrix of constraint equations

Mobility equation to any general mechanism with constant or variable general constraints with simple or multiple joints

$N_{1}, N_{2}$ is the total number of internal and external multiple joints, respectively

$\tilde{n}_{i}, F_{\tilde{n} i} ; n_{i}, F_{n i}$ is the number of links and the mobility of simple joints forming the $i$ th internal and external multiple joints, respectively

Equation of a elementary or a complex (multi loop) mechanisms

$f_{i}^{e}$ is the active mobilities in $i$ th joint

$\lambda_{K}^{e}$ is the dimension of the active motion space

The mobility equation by using the Jacobian matrix of a simple or multi loop closed kinematic chain without exception

(a) A new formula of number of independent loops (b) and (c) are structural formulas as a function of number of branches, platforms and sum of mobility of kinematic pairs and other parameters

(d) Equation for simple structural groups $(\lambda=6,5,4,3,2)$

$c_{b}$ is the total number of branches between mobile platforms 
Table 1 (continued)

\begin{tabular}{|c|c|c|c|}
\hline & Equations & Authors & Commentary \\
\hline & $\begin{array}{l}B \text { is the number of mobile platform; } j_{B} \text { is th } \\
\text { total number of joints on the mobile platfo }\end{array}$ & & \\
\hline 34 & $\begin{array}{l}M=\lambda-\sum_{i=1}^{c_{l}}\left(\lambda-f_{i}\right)\left(\lambda-f_{i}\right) \text { is the degree } \\
\text { of constraint of the platform }\end{array}$ & McCarthy [34] & Mobility equation of a parallel manipulator \\
\hline 35 & $M=(6-d)(l-j-1)+\sum_{i=1}^{j} f_{i}+q$ & Huang and $\mathrm{Li}[35]$ & Structural formula for parallel mechanisms \\
\hline 36 & $\begin{array}{l}\text { (a) } M=\sum_{i=1}^{j} f_{i}-\lambda(c-B) \\
\text { (b) } \sum_{i=1}^{j} f_{i}=\lambda(c-B) \\
\text { (c) } L=c-B, c=c_{l}+c_{b}, c_{l}=j_{B}-2 c_{b}\end{array}$ & $\begin{array}{l}\text { Alizade and } \\
\text { Bayram [36] }\end{array}$ & $\begin{array}{l}\text { (a) Mobility equation of mechanisms } \\
\text { (b) Equations for simple structural groups } \\
\text { (c) New formula of the number of independent loops } \\
c \text { is the sum of legs and branches, } c_{l} \text { is the total } \\
\text { number of legs, connecting mobile } \\
\text { platforms to ground }\end{array}$ \\
\hline 37 & $\begin{array}{l}M=\sum_{i=1}^{j} f_{i}-\sum_{j=1}^{l} S_{j}+S_{\mathrm{p}} \\
S_{\mathrm{p}} \text { and } S_{j} \text { are spatialities of mobile } \\
\text { platform and legs, respectively }\end{array}$ & Gogu [37] & Mobility equation for parallel mechanisms \\
\hline 38 & $\begin{array}{l}\text { (a) } M=(B-c) \lambda+\sum_{i=1}^{j} f_{i}+q-j_{\mathrm{p}} \\
\text { (b) } M=(\lambda+3)+\sum_{l=1}^{c_{l}}\left(d_{l}-D\right) \\
+\sum_{l=1}^{c_{l}}\left(f_{l}-\lambda_{l}\right)+q-j_{\mathrm{p}}, c=c_{l}+c_{b}+c_{h} \\
D \text { is number of dimensions of } \\
\text { vectors in Cartesian space } \\
d_{l} \text { is number of dimensions of } \\
\text { vectors in subspace }\end{array}$ & $\begin{array}{l}\text { Alizade, Bayram } \\
\text { and Gezgin }\end{array}$ & $\begin{array}{l}\text { (a) Mobility equation for robotic systems with } \\
\text { independent loops with variable general constraint } \\
\text { (b) A new structural formula of mobility } \\
\text { loop-legs equation for parallel Cartesian } \\
\text { platform manipulators. } \\
\lambda \text { is the general constraint parameters of } \\
\text { simple structural group } \\
c_{h} \text { is the number of hinges }\end{array}$ \\
\hline
\end{tabular}

$(L=c-B)$ and new formulation for simple structural groups $\left(\sum f_{i}=\lambda(c-B)\right)$, where $c=c_{b}+c_{l}+c_{h}, c_{h}$ is the number of hinges between moving platform, and $c$ is the total number of connections. Also note that, hinges are the revolute pairs that connect mobile platforms to each others. For describing and comparing structural formulas and parameters in structural analysis and synthesis of robotic mechanical system the unique key controlling parameters are used as shown in Table 1.

The basis of structural synthesis of manipulators are based on the principles of truss kinematical unchanging. Determination of indivisible groups as simple structural groups and creating different new manipulators by using their combination had been done by striving to systematize investigation methods of manipulators.

Firstly, Assur [8] developed the concept of the open chain and utilized this concept for plane structure classification. Secondly, the problem of structural synthesis and analysis was investigated by Malushev [38]. The problem of structural synthesis for spatial mechanisms was introduced by Artobolevskii [39]. The task of structural synthesis was solved by using method of developing closed loops. The classes of structural groups are defined by the number of links of the closed loops and the order is equal to the number of legs.

According to the method of structural synthesis that is given by Baranov [40] spatial and plane structural groups have been created from correspond trusses, and class of simple structural groups are defined by the number of closed loops. Prof. Kolchin [41] has introduced concept of passive constraints to account for existence of the paradoxical mechanisms. That concept has not presented any means for identifying the geometric conditions that determine the general constraints. The problem of general constraint parameter was done by Voinea and Atanasiu [42] as the rank of the matrix of coefficients of the unknowns in a system of equations describing the angular velocities of the relative helicoidal movements. Ozol [43] was taken as a straight point in the theory of structural synthesis by the topological property of mechanisms.

The methods of structural synthesis reported by Davies and Crossley [44], Dobrjanskyi [45], Buchsbaym [46], Freudenstein [47], Dobrjanskyi and Freudenstein [48], Manolescu [49] were based on graph theory to find the set of kinematic chains and mechanisms.

The problems of structural analysis and synthesis of plane and spatial structural groups of higher classes were done by Djoldasbekov and Baygunchekov [50] also. Determination of structural groups by using the 
principles of dividing joints and the method of developing joints were done by Dobrovolskii [14] and Kojevnikov [51], respectively.

The structure theory of parallel mechanisms based on the unit of single-open chains was done by Yang [52,53], and the type synthesis of spatial mechanisms on the basis of spatial single loop was introduced by Alizade, Hajiyev and Sandor [54]. The concept of dual graphs and their applications to the automatic generation of kinematic chains was done by Sohn and Freudenstein [55].

A computer-aided method for structural synthesis of spatial manipulators by using method of developing mobile platforms and branches was done by Alizade [36,56]. Class of the structural group is defined by the number of mobile platforms, kind is defined by the set of joints on the mobile platforms, type of the structural group is determined by the number of branches between mobile platforms, and order describes the number of legs that connect mobile platforms to the ground. A computer-aided method for structural synthesis of planar kinematic chains was introduced by Hwang and Hwang [57], and the concept of loop formation which cancels the necessity of the test for isomorphism was also introduced by Rao and Deshmukh [58].

According to the structural synthesis of parallel mechanisms based on the unit of single-open chains, a class of 3 dof ( 3 translation motion), 5 dof ( 3 translation and 2 rotation), and 6 dof ( 3 translation and 3 rotation) parallel robot manipulators were analyzed by Yang et al. [59-61].

The parallel robot manipulators have precise positioning capability, good dynamic performance and high load carrying capacity. However, the 6 dof parallel structures have poor workspace and the direct kinematic solution gives high coupling degree between independent loops. On the other hand, we need to design the given translation and rotation motion of mobile platform. Analysis of research topics mentioned above show that the systematic study of mobility equations of mechanical systems have been described from different points of view, but systematic study of structural synthesis is relatively weak.

Furthermore, this paper enunciates a new structural formula of mobility and new method of designing robot manipulators, the mobile platform that can generate general motion in space, and also generate constraint motion in subspaces. In the meantime, the structural synthesis of serial platform manipulators is identified according to the new equations for simple serial platform structural groups. General guidelines are presented with nine new robot manipulators and tables of serial platform structural groups for designing new several serial platform manipulators.

\section{Structural formula}

An important class of robotic mechanical system consists of parallel platform manipulators, serial platform manipulators, multiple serial chains, and hybrid robotic mechanical systems. One or more grippers can be connected to one or several platforms. That system will describe one or more gripper robotic system. All platform robotic mechanical systems constructed from the actuators and simple structural groups consist of one or more platforms, legs, branches and hinges. Usually actuators are connected to legs. For these robotic mechanical systems loop mobility equations have been used $[25,33,36]$. Other new method of structural synthesis of robot manipulators consists from connecting the simple structural groups to actuators and moving platform. Therefore, if the platform moves in Cartesian system coordinates, simple structural groups will be constructed in the orthogonal planes separately. For these robotic mechanical systems a new loop-legs mobility equation has been used (formulas \#38 in Table 1). In this section, the mobility of these systems are determined. The structural synthesis of serial platform manipulators is based on the structural synthesis of parallel platform manipulators that was described in [36].

Moving platforms that are supported by $c_{l}$ legs, $c_{b}$ branches, and $c_{h}$ hinges, will have the total number dof of joints of the legs as $\sum_{i=1}^{C_{l}} f_{l i}$, branches as $\sum_{i=1}^{C_{b}} f_{b i}$, and the hinges as $\sum_{i=1}^{C_{h}} f_{h i}$, respectively. The total number of legs, branches and hinges gives:

$$
c=c_{l}+c_{b}+c_{h}
$$

And, the total number dof of joints of all legs, branches and hinges would be:

$$
\sum_{i=1}^{C} f_{c i}=\sum_{i=1}^{C_{b}} f_{b i}+\sum_{i=1}^{C_{l}} f_{l i}+\sum_{i=1}^{C_{h}} f_{h i}
$$


All branches, legs and hinges of the manipulators create independent loops as $L_{b}=c_{b}-B+1, L_{l}=c_{l}-1$ and $L_{h}=c_{h}$, respectively, the number of independent loops in closed kinematic chains as shown in [36] can be introduced as:

$$
L=L_{b}+L_{l}+L_{h}=c_{b}+c_{l}+c_{h}-B=c-B
$$

Using Eqs. (1)-(3) we can formulate the following.

\section{Definitions}

- Total number of connection chains is the sum of the number of branches', legs', and hinges.

- Number of independent loops in closed branches' kinematic chains is the difference of the number of branches and platforms plus one.

- Number of independent loops in closed legs' kinematic chains is one less of the number of legs.

- Two platforms that are connected by a hinge will create independent loop.

- Number of independent loops in a closed kinematic chain is the difference of the number of the connection chains and platforms.

Rejoining the moving platforms of these branches, legs, and hinges to form separate $B$ platforms in a space with $\lambda \mathrm{B}$ dof, is the same as removing $L=c-B$ independent loops from the system to form kinematic chains with $\sum_{i=1}^{C} f_{c i}=\sum_{i=1}^{j} f_{i}$. Using structural formula [33], we can describe the mobility loop equation in the following form:

$$
M=\sum_{i=1}^{j} f_{i}-\lambda\left(j_{B}-B-c_{b}\right)+q-j_{\mathrm{p}}=\sum_{i=1}^{C} f_{c i}-\lambda(c-B)+q-j_{\mathrm{p}}=\lambda B+\sum_{i=1}^{C}\left(f_{c i}-\lambda\right)+q-j_{\mathrm{p}}
$$

where, $\lambda B=M_{B}$ is the sum of mobilities of all platforms in the unconstrained space or subspace, and $\sum_{i=1}^{c}\left(f_{i}-\lambda\right)=M_{c}$ is the sum of constraints imposed by the legs, branches and hinges.

Each leg, branch, and hinge separately introduces an insufficient $\left(f_{c i}-\lambda<0\right)$, sufficient $\left(f_{c i}-\lambda=0\right)$, or a redundant $\left(f_{c i}-\lambda>0\right)$ kinematic chain. Sum of degrees of freedom of all platforms and the degrees of constraint that is imposed by kinematic chains describe the mobility of serial platform and parallel platform manipulators.

Mobility loop Eq. (4) for robotic systems with independent loops with variable general constraint could be described as follows:

$$
M=(B-c) \lambda+\sum_{i=1}^{C} f_{c i}+q-j_{\mathrm{p}}
$$

where, $\lambda=2,3,4,5,6$.

Different new platform manipulators could be designed in subspaces $\lambda=2,3,4,5$ and in general space $\lambda=6$.

The aim of the new method of structural synthesis is:

- Using formula (5) we can describe the simple structure groups $(M=0)$ for subspaces $\lambda=2,3,4,5$ and for general space $\lambda=6$ as shown in [36]. A classification of sets of lines linearly dependent on one, two, three, four and five given lines have been introduced by McCarthy notation in [34, pp. 272-282].

- Simple structural groups can be connected to the general moving platform and the actuators that are positioned in the orthogonal Cartesian planes.

- Each actuators will moved (or rotated if it is possible) along the orthogonal Cartesian coordinate system.

Now, our problem is to describe a new structural formula for platform manipulators which operates in Cartesian space or subspace and its legs consist from simple structural groups and actuators operates in orthogonal planes.

Euclidian space geometry introduces that any three vectors that are not on the same plane define a space with dimension $D=3$, also any two non-zero independent vectors define a plane with dimension $d=2$, and in the end, one vector define a line passing through origin of coordinate system $d=1$. 
Let the number of independent parameters describing the structural groups of three legs $c_{l}=3$ that are placed in three orthogonal planes is $\lambda$. The general moving platform and the actuators, positioned along orthogonal axis, are connected by simple structural groups with general constraint parameters $\lambda=3,4,5,6$. Each simple structural group creates the legs and introduces the plane or line with two or one dimensions, thus the total number of leg dimensions are $\sum_{l=1}^{C_{l}} d_{l}$, where $d=1$ or $d=2$. The dimension of constraint of the general moving platform that is imposed by dimensions of each leg can be written as $\sum_{l=1}^{C_{l}}\left(d_{l}-D\right)$. Thus, the motion of the general moving platform in Cartesian orthogonal system will be in the following form:

$$
m_{\mathrm{p}}=(\lambda+3)+\sum_{l=1}^{C_{l}}\left(d_{l}-D\right)
$$

The mobility of legs of the general moving platform is:

$$
M_{l}=\sum_{l=1}^{C_{l}}\left(f_{l}-\lambda_{l}\right)+q-j_{\mathrm{p}}
$$

where $\lambda_{l}$ is the general leg constraint, $f_{l}$ is dof of leg kinematic pairs.

As a result, the mobility of parallel Cartesian platform robot manipulators consist from the motion of the general moving platform $m_{\mathrm{p}}$, and the mobility of legs $M_{l}$ moving in orthogonal planes

$$
M=m_{\mathrm{p}}+M_{l}
$$

Combining the Eqs. (6)-(8) we can describe the new structural formula of mobility loop-legs equations as follows:

$$
M=(\lambda+3)+\sum_{l=1}^{C_{l}}\left(d_{l}-D\right)+\sum_{l=1}^{C_{l}}\left(f_{l}-\lambda_{l}\right)+q-j_{\mathrm{p}}
$$

Example 1. Let us design three parallel Cartesian platform manipulators, where the motion of the general moving platform has translational motions $P z, P y-P z$, and $P x-P y-P z$, respectively.

(A) For the first orthogonal robot manipulator we will take three simple structural groups RRR from the subspace $\lambda=3$, one linear actuator moving along $z$-axis, and for symmetry two links rotating around $x$ and $y$-axes (Table 2.1). In each orthogonal plane, simple structural groups will be connected to the general moving platform, actuator and two rotation links. Using mobility loop-legs Eq. (9) we can calculate the mobility of the type PRR-[RRR]-2RRR parallel orthogonal robot manipulator as: $q=0, j_{\mathrm{p}}=0, \lambda=3, \lambda_{l}=(3,3,4), d_{l}=(1,1,2), \sum f_{l}=12, M=3$. By using Eqs. (6) and (7) the motion of the general moving platform and the mobility of legs will be $m_{\mathrm{p}}=1$ and $M_{l}=2$, respectively.

(B) For the second orthogonal robot manipulator we will take three simple structural groups $\left(n=4, p_{1}=6\right.$, $M=0, \lambda=3$ ) which will be connected to the general moving platform, two linear actuators along $y$ and $z$-axes, and for symmetry one link rotating, around $x$-axis (Table 2.2). Using the same procedure we can calculate the structural parameters of the type 2PR(RRR)[RR-RR-RR]-(RRR)RR parallel orthogonal robot manipulator as: $M=3, m_{\mathrm{p}}=2, M_{l}=1$. Note that the mobility of each leg will be calculated from two loops as: $M_{l}=[(4-3)+(3-4)]+[(4-3)+(3-4)]+[(4-3)+(3-3)]=1$.

(C) For the third orthogonal manipulator we will follow the same steps in Example 1(B) except three actuators will be used along the $x, y$, and $z$-axes (Table 2.3). The structural parameters for $3 \mathrm{PR}(\mathrm{RRR})[\mathrm{RR}$ RR-RR] parallel orthogonal manipulator will be $M=3, m_{\mathrm{p}}=3, M_{l}=0$.

Example 2. Let us design two parallel Cartesian platform manipulators, where, in first, the motion of the general moving platform has three rotational motions $R x, R y, R z$ and one translational motion on the line in $x-y$ plane $(P x y)$ and in second, the motion of the general moving platform has one rotational motion $R z$ and three translational motions $P x, P y$ and $P z$.

(A) For the first orthogonal robot manipulator we will take three simple structural groups RRRR from the subspace $\lambda=4$, and three linear actuators moving along $x, y$ and $z$-axes (Table 2.4). In each orthogonal 
Table 2

New parallel Cartesian robot manipulator types

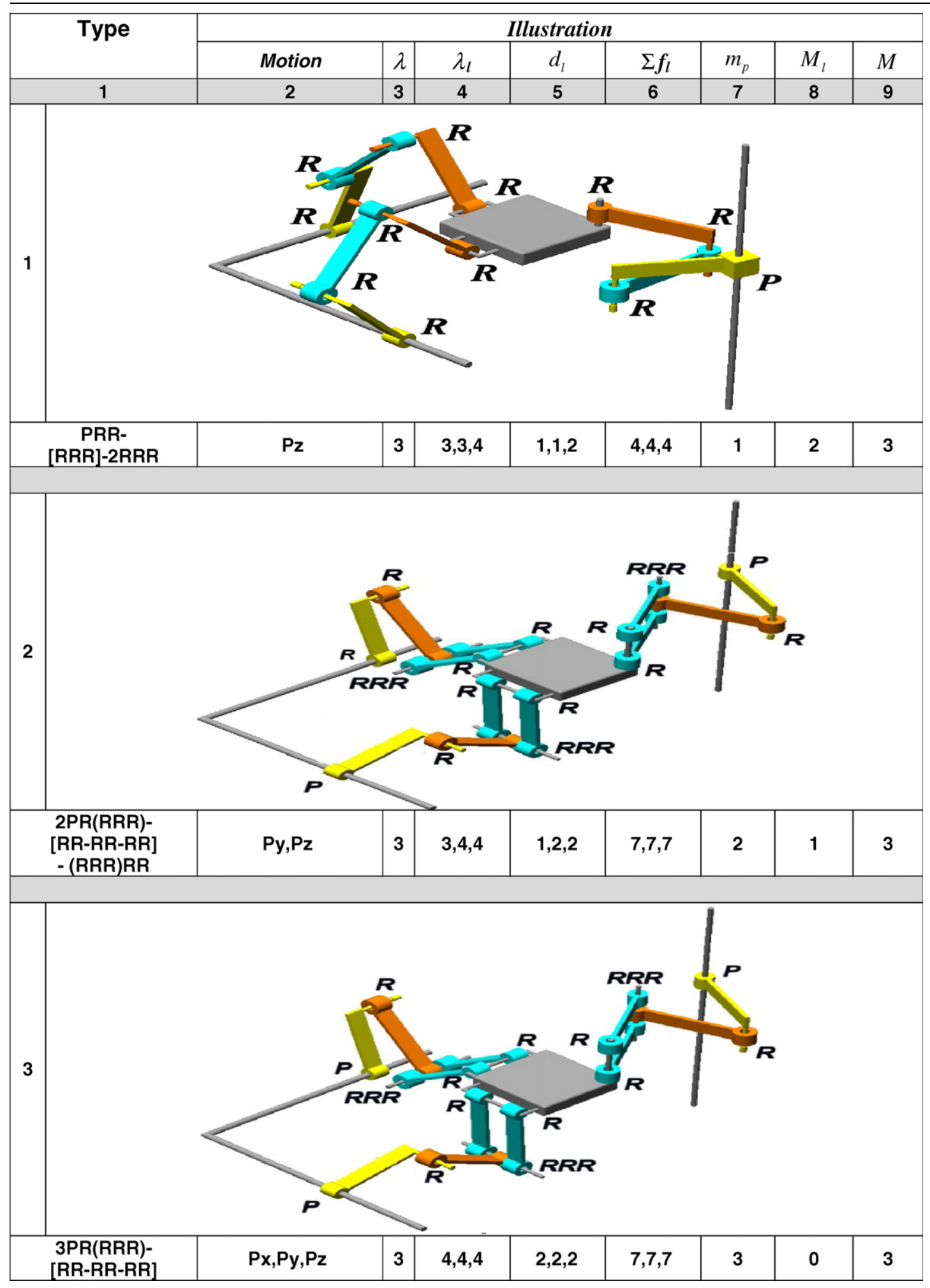


Table 2 (continued)

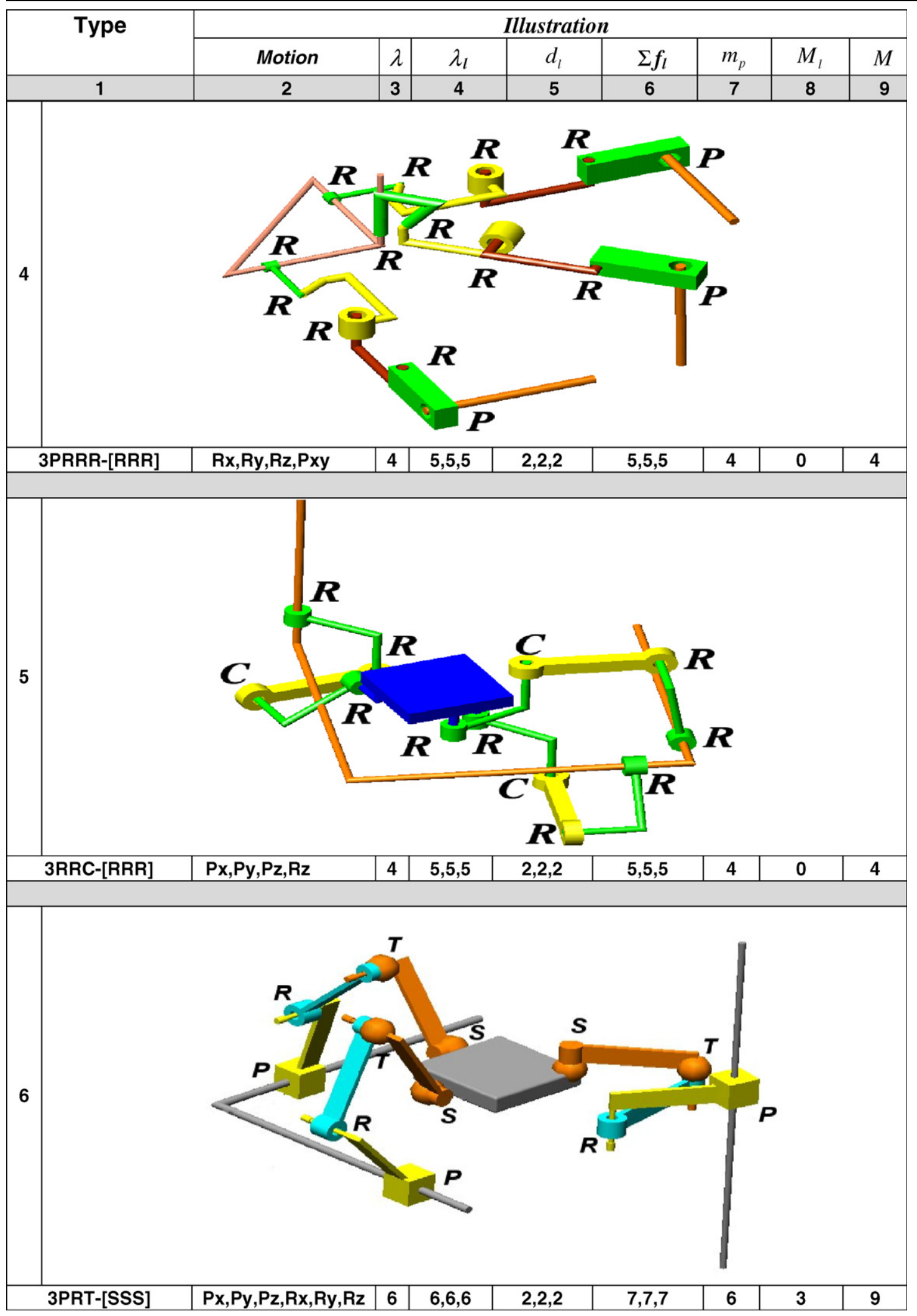


Table 2 (continued)

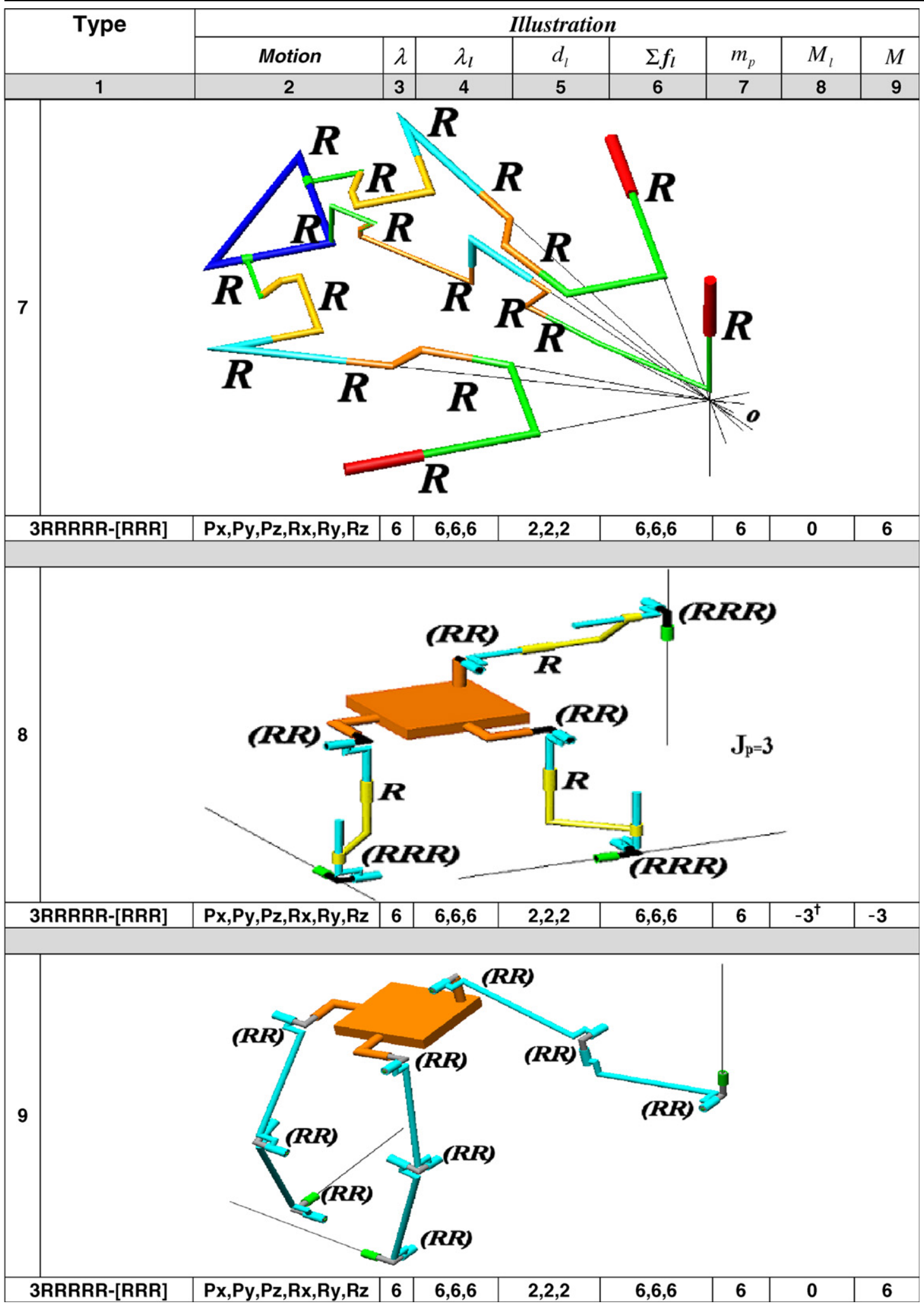

${ }^{\dagger} M_{l}=-3$ comes from the passive degrees of freedom, $j_{p}=3$. 
plane, simple structural groups will be connected to the general moving platform and actuators. Using mobility loop-legs Eqs. (9), (7) and (6) we can calculate the structural parameters of the type 3PRRR[RRR] parallel orthogonal robot manipulator as: $M=4, m_{\mathrm{p}}=4, M_{l}=0$. Note that to reach the given motion of the general moving platform we need to add one more actuator.

(B) For the second orthogonal robot manipulator we will take three simple structural groups RCR from the subspace $\lambda=4$, which will be connected to the general moving platform, three rotational actuators in $x$, $y$ and $z$-axes (Table 2.5). Using the same procedure we can calculate the structural parameters of the type $3 \mathrm{RRC}[\mathrm{RRR}]$ parallel orthogonal robot manipulator as: $M=4, m_{\mathrm{p}}=4, M_{l}=0$. We need additional one actuator to reach the given motion of the general moving platform.

Example 3. Let us design a parallel Cartesian platform manipulator, where the motion of the general moving platform has three rotational motions $R x, R y, R z$ and three translational motions $P x, P y$ and $P z$. First we will take three simple structural groups STR from the space $\lambda=6$, and three linear actuators moving along $x, y$ and $z$-axes (Table 2.6). In each orthogonal plane, simple structural groups will be connected to the general moving platform and actuators. Using mobility loop-legs Eqs. (9), (7) and (6) we can calculate the structural parameters of the type 3PRT[SSS] parallel orthogonal robot manipulator as: $M=9, m_{\mathrm{p}}=6, M_{l}=3$. Note that to reach the given motion of the general moving platform we need to add six more actuators. Also to get rid of excessive mobility $\left(M=6, m_{\mathrm{p}}=6, M_{l}=0\right)$ we can connect each $\lambda=6$ structural group directly to the $x$, $y$ and $z$-axes (Table 2.6-2.7-2.9).

Note: Due to the fact that, using the same analogy, in all our trials with $\lambda=5$ structural groups, the legs of the parallel Cartesian platform manipulators are converted to $\lambda=6$ structural groups, the motion of the manipulators is transformed into $R x, R y, R z, P x, P y$ and $P z$ (Table 2.6-2.9). So that investigation for $m_{\mathrm{p}}=5$ with $\lambda=5$ structural groups will be continued in future.

\section{Structural synthesis and classification of simple serial platform structural groups}

Serial platform kinematic chains means that, at least two platforms are connected by hinge kinematic pairs (and, therefore, zero number of branches as well) and all legs are going from the mobile platforms to the frame.

The problem of creating simple structural groups for plane and spatial serial platform kinematic chains is considered by developing platforms and closed loops. Simple serial platform structural group is the one that cannot be splitted into several other structural groups with smaller number of links. A simple serial platform structural group have the mobility equal to zero $(M=0)$, thus the number of input parameters are zero.

The plane simple structural groups can be created by lower and higher kinematic pairs, and spatial structural groups by hinge, revolute, spheric and slotted spheric kinematic pairs. Using exchangeability of kinematic pairs we can describe different structure of simple structural group (and, therefore, the hinge joint between mobile platforms is not changed, as well).

For creating simple structural platform structural groups, mobility loop Eq. (5) could be described as follows:

$$
(B-c) \lambda+\sum_{i=1}^{c} f_{c i}=0
$$

where $c=c_{l}+c_{h}$, as $c_{b}=0$.

Simple structural group Eq. (9) for subspace $\lambda=3$, and for general space $\lambda=6$ can be introduced, respectively as:

$$
\begin{aligned}
& \sum_{i=1}^{c_{l}+c_{h}} f_{c i}=3\left(c_{l}+c_{h}-B\right) \\
& \sum_{i=1}^{c_{l}+c_{h}} f_{c i}=6\left(c_{l}+c_{h}-B\right)
\end{aligned}
$$


Table 3

Simple structural groups of serial platform manipulators in subspace $\lambda=3$

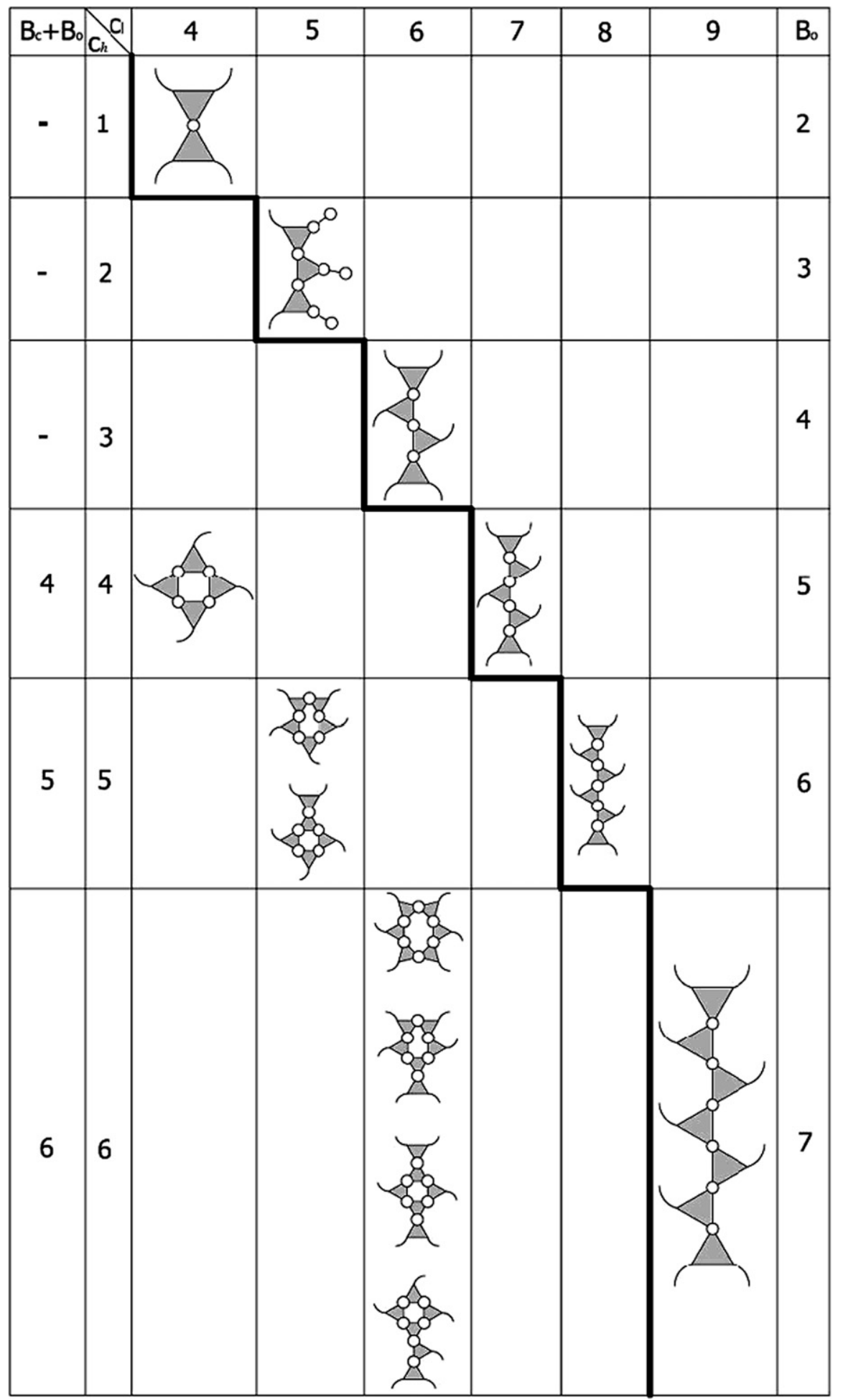


Table 4

Simple structural groups of serial platform manipulators in space $\lambda=6$

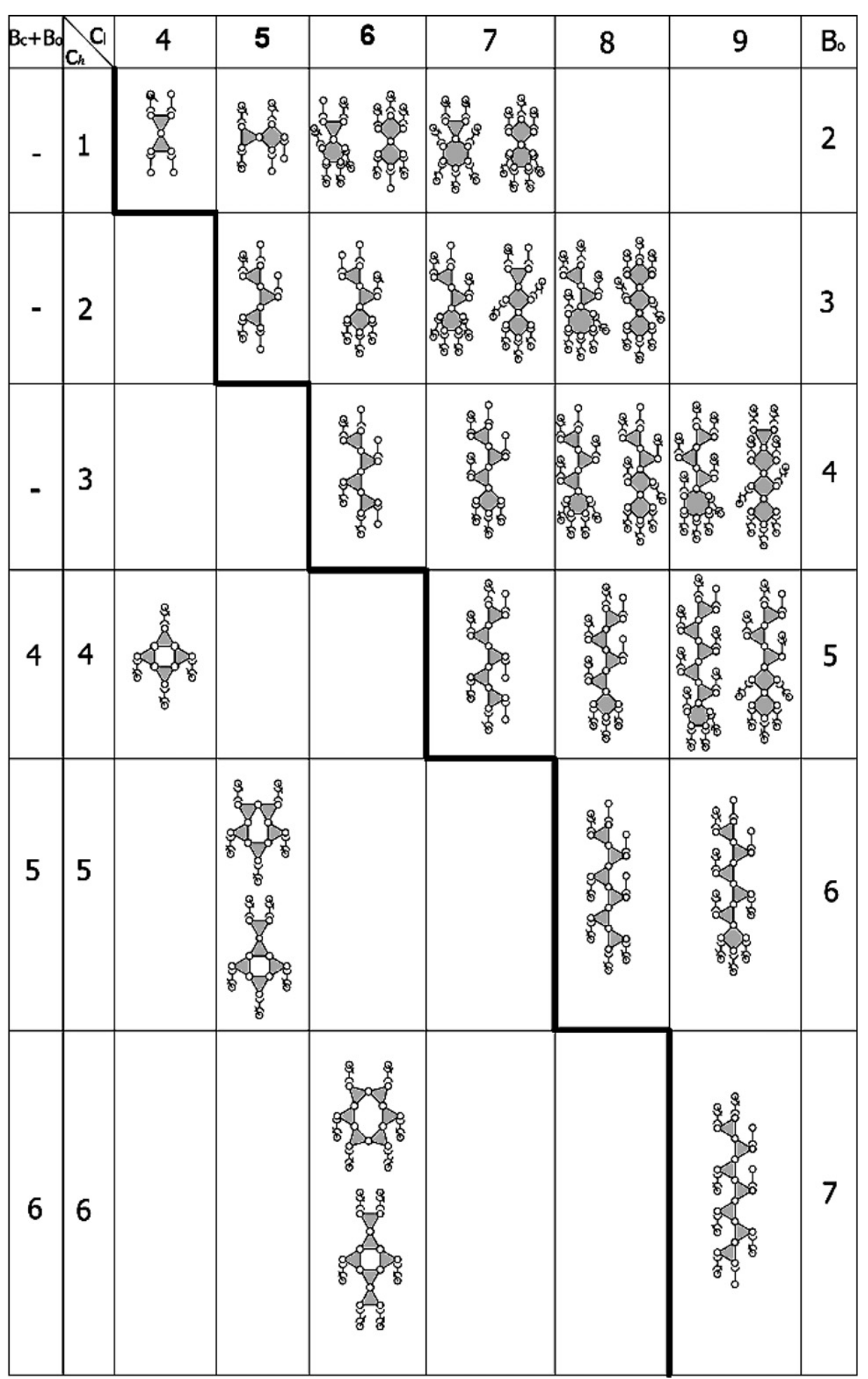


The additional conditions of structural synthesis of serial platform kinematic chains can be introduced as following equalities and inequalities:
(a) $L=c_{l}+c_{h}-B$
(b) $c_{l}=j_{B}-2 c_{h}$
(c) $j=3 L, j=6 L$
(d) $j_{l}=j / c_{l}$
(e) $3 \leqslant j_{B} \leqslant 6$
$(f) c=c_{l}+c_{h}$
(g) $B \geqslant 2$

Using objective functions (10)-(12) and additional equality and inequality constraint conditions (13), computer software of structural synthesis of simple serial platform structural groups has been created. Results of plane and spatial simple serial structural groups are presented in the following Tables 3 and 4 .

The algorithm of structural synthesis of serial platform simple structural groups can be summarized step by step as follows:

- Take subspace $\lambda=3$, or general space $\lambda=6$.

- Select values for $B$ and $j_{B}$ Eqs. (13e) and (13g).

- Select value for hinge joints $\mathrm{c}_{h}$ and calculate the number of legs $\mathrm{c}_{l}$ Eq. (13b).

- Calculate the number of independent loops $L$ Eq. (13a).

- Calculate the number of joints $j$ with one dof Eq. (13c).

- Place the joints on legs Eq. (13d) and selected hinge joints $\mathrm{c}_{h}$ between mobile platforms.

- Using the principle of exchangeability of kinematic pair, replace the joints with one dof with higher and other kinematic pairs.

- The mobility of manipulator is equal to the number of actuators (Eq. (5)) added to the legs of simple serial platform structural group.

The simple serial platform structural groups in subspace $\lambda=3$ and in general space $\lambda=6$ has been introduced by serial platform kinematic chains with open loops $B_{o}$, closed loops $B_{c}$, and mixed open and closed loops $\mathrm{B}_{\mathrm{o}}+\mathrm{B}_{\mathrm{c}}$, as shown in Tables 3 and 4 , respectively.

Example. To design spatial robot manipulator with 6 dof and two grippers placed on two mobile platforms. Select from Table 4 simple serial platform structural group with $\mathrm{c}_{l}=6$, and $\mathrm{c}_{h}=1$, which consist from two mobile platforms $B=2$, and one hinge joint $\mathrm{c}_{h}=1$, thus $c=c_{l}+c_{h}=7$ and joints on triangular platform $j_{B 1}=3$, and on pentagonal platform $j_{B 2}=5$, and $j_{B}=j_{B 1}+j_{B 2}=8$. The number of joints of simple structural group is $\sum f_{i}=30$. Six actuators will be placed on six legs and spherical, prismatic, and revolute pairs are used

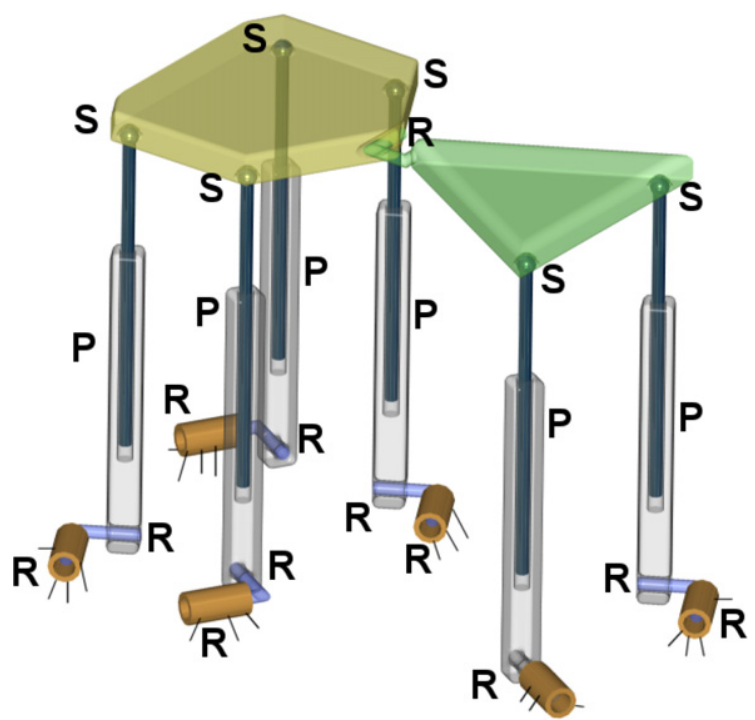

Fig. 1. 6 dof spatial serial platform robot manipulator. 
in this structure. Using Eq. (5) gives, $M=(B-c) \lambda+\sum_{i=1}^{C} f_{c i}=(2-7) 6+36=6$. The structure of serial platform robot manipulator is introduced in Fig. 1.

\section{Structural synthesis parallel cartesian platform robot manipulators}

Kinematic chain shown in Table 5.1 is referred as a simple Cartesian structural group. Simple Cartesian structural group can be obtained by successive coupling of the three simple structural groups in the orthogonal planes

Table 5

Variations of actuators for simple structural group RRR $(\lambda=3)$ of parallel Cartesian platform robot manipulators

\begin{tabular}{|c|c|c|c|c|c|c|c|}
\hline & Type & $\overline{d_{i}}$ & $\lambda_{l}$ & $\overline{f_{l}}$ & $m_{\mathrm{p}}$ & $M_{l}$ & $\bar{M}$ \\
\hline 1 & 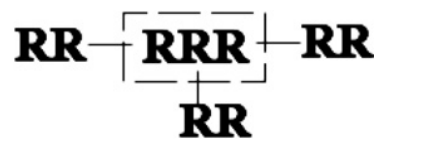 & $1,1,1$ & $3,3,3$ & $3,3,3$ & 0 & 0 & 0 \\
\hline 2 & 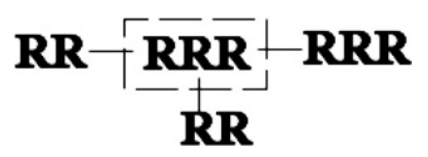 & $1,1,1$ & $3,3,3$ & $3,3,4$ & 0 & 1 & 1 \\
\hline 3 & $\underset{\mathbf{R R}}{-\mathbf{R R}_{\mathbf{R}}-\mathbf{R R P}}$ & $1,1,2$ & $3,3,4$ & $3,3,4$ & 1 & 0 & 1 \\
\hline 4 & 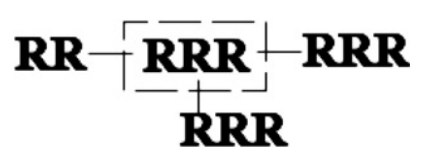 & $1,1,1$ & $3,3,3$ & $3,4,4$ & 0 & 2 & 2 \\
\hline 5 & $\mathbf{R R} \underbrace{\mathbf{R R}}_{\mathbf{R} \mathbf{R}}-\mathbf{R R P}$ & $1,1,2$ & $3,3,4$ & $3,4,4$ & 1 & 1 & 2 \\
\hline 6 & 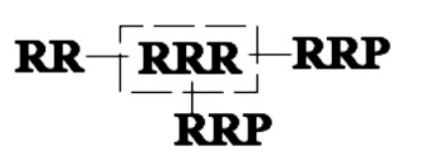 & $1,2,2$ & $3,4,4$ & $3,4,4$ & 2 & 0 & 2 \\
\hline 7 & 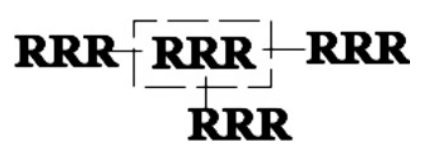 & $1,1,1$ & $3,3,3$ & $4,4,4$ & 0 & 3 & 3 \\
\hline 8 & 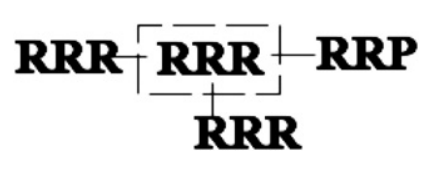 & $1,1,2$ & $3,3,4$ & $4,4,4$ & 1 & 2 & 3 \\
\hline 9 & 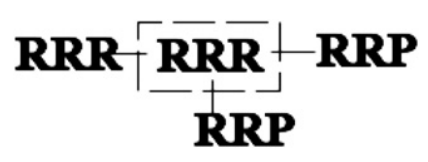 & $1,2,2$ & $3,4,4$ & $4,4,4$ & 2 & 1 & 3 \\
\hline 10 & 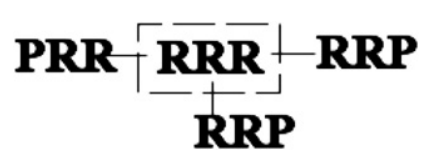 & $2,2,2$ & $4,4,4$ & $4,4,4$ & 3 & 0 & 3 \\
\hline
\end{tabular}


to the general moving platform. Thus, simple Cartesian structural group is one of the orthogonal parallel groups that cannot be split into several orthogonal parallel structural groups with smaller members of links. A simple Cartesian structural group has zero number of mobility (Eq. (9)), that is number of input links equals to zero.

$$
(\lambda+3)+\sum_{l=1}^{C_{l}}\left(d_{l}-D\right)+\sum_{l=1}^{C_{l}}\left(f_{l}-\lambda_{l}\right)=0
$$

Such structural group can be reached from Eq. (14) if and only if the motion of the general moving platform $m_{\mathrm{p}}=0$ and the leg mobility $M_{l}=0$ :

$$
\begin{aligned}
& (\lambda+3)+\sum_{l=1}^{C_{l}}\left(d_{l}-D\right)=0 \\
& \sum_{l=1}^{C_{l}}\left(f_{l}-\lambda_{l}\right)=0
\end{aligned}
$$

The kinematic chain shown in Table 5.1 is the first simple Cartesian structural group that was constructed in each orthogonal plane by simple structural group RRR, with the general constraint parameter $\lambda_{l}=3$, and the number of dimension $d=1$.

The result of the generation principle of parallel Cartesian platform robot manipulators is shown in Table 5. Every Cartesian robot manipulator was generated by the successive joining of orthogonal simple structural groups to the actuators on the orthogonal frames. Table 5.2, 5.4, and 5.7 shows Cartesian robots with mobility of legs $M_{l}=1,2,3$ (Eq. (16)), motion of the general moving platform $m_{\mathrm{p}}=0$ (Eq. (15)), and mobility of robot manipulator $M=1,2,3$ (Eq. (14)), respectively.

The generation of a parallel Cartesian platform robot manipulator with one motion of the general moving platform $m_{\mathrm{p}}=1$ can be generated by three kinematic chains as was shown in Table 5.3, 5.5 and 5.8. The mobilities of legs are $M_{l}=0,1$ and 2, and the mobilities of manipulators are $M=1,2$, and 3, respectively.

Table 5.6 and 5.9 indicates the two motions of the general moving platform $m_{\mathrm{p}}=2$ with other parameters as $M_{l}=0,1$ and $M=2,3$. The generation of the Cartesian robot manipulator with three motion of the general moving platform $m_{\mathrm{p}}=3$, mobility of legs $M_{l}=0$, and the mobility $M=3$ with three linear actuators moving along the orthogonal axes was shown in Table 5.10.

In the end, generation of the variations of actuators for simple structural group $\operatorname{RRR}(\lambda=3)$ in the orthogonal planes gives five parallel Cartesian platform robot manipulators (Table 5.5, 5.6, 5.8-5.10).

\section{Conclusions}

A new structural formula for spatial parallel manipulators having one general moving platform, working in Cartesian space, having three legs that are placed in orthogonal planes introducing simple structural groups in subspaces $\lambda=3,4$ and general space $\lambda=6$, and connected to actuators and to the general moving platform is introduced. History of formulas dof is presented as 38 equations with the unique key controlling parameters. Nine new parallel Cartesian platform robot manipulators are introduced by applying new mobility loop-legs equation. Structural synthesis of serial platform manipulators and parallel Cartesian platform manipulators are considered. Simple serial platform structural groups in subspace $\lambda=3$, and general space $\lambda=6$ are presented along with examples.

\section{References}

[1] R. Courant, H. Robbins, I. Stewart, 1996, What is mathematics, Euler's formula, NY, USA, 1752, pp. 235-240.

[2] P.L. Chebyshev, Théorie des mécanismes connus sais le non de parallélogrammes, 2 éme partie, Mémoires présentes a 1'Academie impériale des sciences de Saint-Pétersbourg par divers savants, 1869.

[3] J.J. Sylvester, On recent discoveries in mechanical conversion of motion, Proc. R. Inst. Great Britain 7/5 (1874) $179-198$.

[4] M. Grübler, Allgenmeine Eigenschaften der swanglaufigen ebeden kinematischen ketten part I, Zivilingenieur 29 (1883) 167-200; Part II, Verh. Ver. Bef. Gew. 64 (1885) 179-223.

[5] P.O. Somov, On the dof of kinematic chains, J.P. Chen 19 (9) (1987) 443-447. 
[6] Kh.I. Gokhman, Equation for mobility definition and solution of mechanism classification, Odessa, 1889, pp. 24-50.

[7] G. Koenigs, Introduction a une théorie nouvelle des mécanismes, Librairie Scientifique A. Hermann, Paris, 1905, pp. 27-28.

[8] L.V. Assur, Investigation of plane linkage mechanisms with lower pairs from point of view of their structure and classification, unpublished dissertation (1916), AS USSR (1952) 529.

[9] R. Mueller, Die zwanglanfigkeit kinematische ketten, Unpublished dissertation (1920), as quoted in K. Federhofer: Graphische Kinematic and Kinetostatic, Springer, Berlin, 1932.

[10] A.P. Malushev, Analysis and synthesis of mechanisms from point of view of their structure, Tomsk (1923) 91

[11] K. Kutzbach, Mechanische leitungsverzweigung, ihre gezetze und anwendungen, Maschinenbau. Betrieb 8 (1929) $710-716$.

[12] N.I. Kolchin, Applied mechanic in problems, issue I-IV, Peterburg, 1932-1934.

[13] I.I. Artobolevskii, To structure of spatial mechanisms, 10 (1935) 110-152; Experience of structural analysis, Structure and classification of mechanisms, AS USSR Moscow (1939) 49-66.

[14] V.V. Dobrovolskii, Main principles of rational classification, AS USSR (1939) 5-48.

[15] U.F. Moroshkin, Geometry problems of complex kinematic chains, AS USSR 119 (1) (1958) 38-41.

[16] R. Voinea, M. Atanasiu, Contributions a la theorie geometrigue des vis, Bull. Inst. Politech. Bucuresti XXI (3) (1959).

[17] B. Paul, A unified criterion for the degree of constraint of plane kinematic chains, J. Appl. Mech. (27) (1960) 196-200.

[18] W. Rössner, Zur strukturellen ordnung der getriebe, Wissenschaft. Tech. Univ. Dresden 10 (1961) 1101-1115.

[19] H. Boden, Zum zwanglauf genuscht raümlichebener getriebe, Maschinenbautechnic (11) (1962) 612-615.

[20] O.G. Ozol, A new structural formula for mechanisms and its theoretical and practical importance, Latv. Agric. Acad. (11) (1962) 113129.

[21] K.J. Waldron, The constraint analysis of mechanisms, J. Mech. 1 (1966) 101-114.

[22] N. Manolescu, For a united point of view in the study of the structural analysis of kinematic chains and mechanisms, J. Mech. 3 (1968) 149-169.

[23] C. Bagci, Degrees of freedom of motion in mechanisms, ASME J. Eng. Ind. 93B (1971) 140-148.

[24] P. Antonescu, Extending of structural formula of Dobrovolski to the complex mechanisms with apparent family, in: Proceedings of the SYROM, Bucharest, 1973.

[25] F. Freudenstein, R. Alizade, On the degree of freedom of mechanisms with variable general constraint, in IV World IFToMM Congress, England, 1975, pp. 51-56.

[26] K.H. Hunt, Kinematic Geometry of Mechanisms, Oxford Univ. Press, Oxford, 1978.

[27] J.M. Herve, Analyse structurelle des mécanismes par groupe des déplacements, Mech. Mach. Theory 13 (1978) $437-450$.

[28] A. Gronowicz, Identifizierungs-Methode der Zwanglaufbedingungen von kinematischen ketten, Elsevier IFToMM, J. Mech. Mach. Theory 16 (1981) 127-135.

[29] T.H. Davies, Kirchoff's circulation law applied to multi-loop kinematic chains, Elsevier IFToMM, J. Mech. Mach. Theory 16 (1981) 171-183.

[30] V.P. Agrawal, J.S. Rao, Fractionated freedom kinematic chains and mechanisms, Elsevier IFToMM, J. Mech. Mach. Theory 22 (1987) 125-130.

[31] F. Dudita, D. Diaconescu, Optimizarea structurala a mecanismelor, Technica Bucuresti (1987) 36-45, see also pages $229-254$.

[32] J. Angeles, C. Gosselin, Determination du degree de liberte des chaines cinematiques, Trans. CSME 12 (4) (1988) $219-226$.

[33] R.I. Alizade, On degree of freedom of kinematic chain, Azerbaijan Polytech. Inst. Automation design of mechanisms, manipulators and robots, Baku (1988) 3-14.

[34] J.M. McCarthy, Geometric Design of Linkages, Springer Verlag, New York, 2000, p. 320.

[35] Z. Huang, Q.C. Li, Type synthesis of symmetrical lower-mobility parallel mechanisms using the constraint-synthesis method, J. Robot. Res. 22 (2003) 59-79.

[36] Rasim Alizade, Cagdas Bayram, Structural synthesis of parallel manipulators, Elsevier, IFToMM, J. Mech. Mach. Theory 39 (2004) 857-870.

[37] Grigore Gogu, Mobility of mechanisms: a critical review, Elsevier, IFToMM, J. Mech. Mach. Theory 40 (2005) $1068-1097$.

[38] A.P. Malushev, Analysis and synthesis of mechanisms from point of view of their structure, Tomsk (1929) 78.

[39] I.I. Artobolevskii, Structural analysis experience of mechanisms, Structure and classification of mechanisms, AS USSR (1939) 49-66.

[40] G.G. Baranov, Classification, structure, kinematic and kinetostatic of mechanisms with first order pairs, AS USSR, Mech. Mach. Theory 2 (46) (1952) 15-39.

[41] N.I. Kolchin, Experience structure of widening structural classification of mechanisms and base to its structural table of mechanisms, Anal. Synth. Mech. Moscow (1960) 85-97.

[42] R. Voinea, M. Atanasiu, Contribution a l'etude de la structure des chaines cinematiques, Bulrtinul Institutului Pol. Bucharesti 21 (1) (1960).

[43] O.G. Ozol, Expansion of structural theory and classification plane mechanisms with lower pairs, Latv. Acrycult. Acad. (13) (1963) 7191.

[44] T.H. Davies, F.R.E. Crossley, Structural analysis of plane linkages by Franke's condensed notation, J. Mech. 1 (1966) 171-183.

[45] L. Dobrjanskyi, Application of graph theory to the structural classification of mechanisms, Ph.D. Disertation, Columbia University, 1966.

[46] F. Buchsbaym, Structural classification and type synthesis of mechanisms with multiple elements, Ph.D. Disertation, Columbia University, 1967.

[47] F. Freudenstein, The basic concepts of Polya's theory of enumeration with application to the structural classification of mechanisms, J. Mech. 3 (2) (1967) 275-290. 
[48] L. Dobrjanskyi, F. Freudenstein, Some applications of graph theory to the structural analysis of mechanisms, Trans. ASME J. Eng. Ind. (1967) 153-158.

[49] N.I. Manolescu, A method based on Baranov trusses and using graph theory to find the set of planar jointed kinematic chains and mechanisms, Elsevier, IFToMM, J. Mech. Mach. Theory (1) (1973) 3-22.

[50] Y.A. Djoldasbekov, J.J. Baygunchekov, Structural analysis one loop Assur groups of higher class, Kaz. Acad. Sci. (12) (1976).

[51] S.N. Kojevnikov, Foundation of structural synthesis of mechanisms, Kiyev (1979) 229.

[52] T. Yang, Structural analysis and number synthesis of spatial mechanisms, Sixth World Congress on Theory Mach. and Mech., New Delhi, India, 1983, pp. 280-283.

[53] T. Yang, Structural synthesis of spatial multi loop chains with variable over constraints, The Fourth IFToMM Symposium, Bucharest, Romania, vol. 11 (2), 1985, pp. 447-456.

[54] R.I. Alizade, E.T. Hajiyev, G.N. Sandor, Type synthesis of spatial mechanisms on the basis of spatial single loop structural groups, IFToMM, J. Mech. Mach. Theory 20 (2) (1985) 95-101.

[55] W.J. Sohn, F. Freudenstein, An application of dual graphs to the automatic generation of kinematic structures of mechanisms, Trans. ASME 108 (1986) 392-398.

[56] R.I. Alizade, Investigation of linkage mechanisms with lower pairs from point of view of its structure and classification, Azerb. Poly. Inst. Baku (1988) 111-127.

[57] W.M. Hwang, Y.W. Hwang, Computer-aided structural synthesis of mechanisms, Trans. ASME 108 (1986) $392-398$.

[58] A.C. Rao, P.B. Deshmukh, Computer aided structural synthesis of planar kinematicchains with simple joints, Elsevier, IFToMM, J. Mech. Mach. Theory 30 (1995) 1193-1215.

[59] Q. Jin, T. Yang, et. al. Structure synthesis of a class of five dof (three translation and two rotation) parallel robot mechanisms based on single-opened-chain units, in: Proceedings of ASME 27th design Automotion Conference, Pittsburgh, DETC/DAC-21153, 2001.

[60] Q. Jin, T. Yang, et al., Structure synthesis of a class of three dof translational parallel robotic mechanisms based on the units singleopened-chain, Chin. J. Mech. Eng. 38 (8) (2002).

[61] H. Shen, T. Yang, L. Ma, Synthesis and structure analysis of kinematic structures of 6 dof parallel robotic mechanisms, Elsevier, IFToMM J. Mech. Mach. Theory 40 (2005) 1164-1180. 